Pacific Journal of Mathematics

MODULES SATISFYING ACC ON A CERTAIN TYPE O 


\title{
MODULES SATISFYING ACC ON A CERTAIN TYPE OF COLONS
}

\author{
Chin-Pi LU
}

\begin{abstract}
Let $M$ be a module over a ring $R$, which satisfies the ascending chain condition on submodules of the form $N: B \subseteq N: B^{2} \subseteq N: B^{3} \subseteq$ ... for every submodule $N$ of $M$ and every finitely generated ideal $B$ of $R$. We investigate the class of such modules $M$ and show that various important properties of Noetherian modules and rings can be generalized to modules and rings of this class.
\end{abstract}

Introduction. Let $M$ be a module over a ring $R$ (commutative with identity). $M$ is said to satisfy (accr) (resp. (accr*)) if the ascending chain of residuals of the form $N: B \subseteq N: B^{2} \subseteq N: B^{3} \subseteq \cdots$ terminates for every submodule $N$ of $M$ and every finitely generated (resp. principal) ideal $B$ of $R$. The class of modules satisfying (accr) is large. It contains Noetherian modules, modules over Artinian rings, modules having ACC on colon submodules ([14]), Laskerian modules, modules over perfect rings, etc. The purpose of this paper is to investigate this class of modules and show that these modules enjoy various important properties of Noetherian modules.

In $§ 1$, we prove that (accr) and (accr*) are equivalent properties of modules. We also characterize modules satisfying this property as those $R$-modules $M$ in which every submodule $N$ can be written in the form $N=\left(N: B^{h}\right) \cap\left(N+B^{h} M\right)$ for every finitely generated ideal $B$ of $R$ and for all sufficiently large positive integers $h$.

Section 2 deals with fundamental properties of modules satisfying (accr). The main result is that both a weak version of the Artin-Rees Lemma and the Krull Intersection Theorem for Noetherian modules can be generated to modules with (accr). We also give an example of a module in order to show that every Laskerian module satisfies (accr), but a module satisfying (accr) is not necessarily Laskerian.

In §3, we consider conditions under which modules (resp. quasi-semilocal rings) satisfying (accr) are Laskerian (resp. Noetherian). Let $M$ be a module each of whose factor module is finite dimensional [10]. We prove that such an $R$-module $M$ is Laskerian $\Leftrightarrow M$ satisfies (accr) $\Leftrightarrow$ the ArtinRees property holds for every submodule $N$ of $M$ and every principal ideal ( $r$ ) of $R$, that is $N \cap r^{h} M \subseteq r N$ for all sufficiently large integers $h$. 
Section 4 is concerned with topological rings satisfying (accr). Applying some topological observations, we prove that both zero-dimensional rings and one-dimensional domains satisfy (accr). It is well-known that if $R$ is a Noetherian ring and $R^{*}$ is the completion of $R$ for the separated $\left(b_{1}, b_{2}, \ldots, b_{n}\right)$-adic topology, then

$$
R^{*} \cong R\left[\left[x_{1}, x_{2}, \ldots, x_{n}\right]\right] /\left(x_{1}-b_{1}, x_{2}-b_{2}, \ldots, x_{n}-b_{n}\right) .
$$

We show that this is also the case for rings of dimension zero.

In $\S 5$, we emphasize that if $M$ is an $R$-module satisfying (accr) and $B$ is a finitely generated ideal of $R$ contained in the Jacobson radical of $R$, then every submodule of $M$ is closed for the $B$-adic topology. Thus $M$ behaves like a Noetherian module over a Zariski ring. Our main purpose in this section is to study topological coherent rings which satisfy (accr). We find that such rings $R$ are also similar to topological Noetherian rings. For example, if $B=\left(b_{1}, b_{2}, \ldots, b_{n}\right)$ is an ideal of $R$, then the Hausdorff completion $R^{*}$ of $R$ for the $B$-adic topology is a flat $R$-module and $R^{*}$ is a faithfully flat $R$-module iff $B$ is contained in the Jacobson radical of $R$; in the latter case we have that

$$
R^{*} \cong R\left[\left[x_{1}, x_{2}, \ldots, x_{n}\right]\right] /\left(x_{1}-b_{1}, x_{2}-b_{2}, \ldots, x_{n}-b_{n}\right) .
$$

Every ring in this paper is a commutative ring with identity and every module is unitary. For definitions not given in the paper the reader is referred to [13].

1. Modules satisfying (accr). Let $M$ be a module over a ring $R, N$ a submodule of $M$, and $B$ an ideal of $R$. The residual $N: B$ of $N$ by $B$ is also called a colon submodule [14]. In this section, we consider exclusively those ascending chains of colon submodules which are of the form $N: B \subseteq N: B^{2} \subseteq N: B^{3} \subseteq \cdots$.

Lemma 1. Let $M$ be an $R$-module, $r \in R$, and $n \in Z^{+}$, the set of positive integers. Then the following statements are equivalent:

(1) The ascending chain of submodules $\left\{(0): r^{k}\right\}_{k \in Z^{+}}$stops at $n$;

(2) $(0): r^{n}=(0): r^{n+1}$;

(3) $\left((0): r^{n}\right) \cap r^{n} M=(0)$.

The proof of Lemma 1 is straightforward, hence we omit it.

Proposition 1. Let $N$ be a submodule of an $R$-module $M$ and $r \in R$. Then the following statements are equivalent:

(1) The ascending chain of submodules $\left\{N: r^{k}\right\}_{k \in Z^{+}}$terminates;

(2) There exists an $n \in Z^{+}$such that $N: r^{n}=N: r^{n+1}$;

(3) There exists an $n \in Z^{+}$such that $N=\left(N: r^{h}\right) \cap\left(N+r^{h} M\right)$ for every $h \geq n$. 
Proof. We apply Lemma 1 to the factor module $M / N$ over $R$.

We remark that, although the proofs are not trivial, both Lemma 1 and Proposition 1 remain valid if we replace $r \in R$ with any finitely generated ideal of $R$. We shall prove this statement later in Corollary to Theorem 1.

If $M$ is a module over $R$ in which (0) is a primary submodule, then every zero divisor of $M$ is nilpotent modulo Ann $M$. Therefore, we can see easily that the ascending chain of submodules $\left\{(0): r^{k}\right\}_{k \in Z^{+}}$terminates for every $r \in R$. Applying this to factor modules of $M$ over $R$, we have

Proposition 2. If $N$ is a primary submodule of an $R$-module $M$, then the ascending chain of submodules $\left\{N: r^{k}\right\}_{k \in Z^{+}}$terminates for every $r \in R$. Consequently, if $L$ is the intersection of a finite number of primary submodules, then the sequence $\left\{L: r^{k}\right\}_{k \in Z^{+}}$terminates for every $r \in R$.

Definition 1. A module $M$ over a ring $R$ is said to satisfy (accr) (resp. (accr*)) if for every submodule $N$ of $M$ and every finitely generated (resp. principal) ideal $B$ of $R$, the ascending chain of residuals $\left\{N: B^{k}\right\}_{k \in Z^{+}}$terminates. A ring satisfies (accr) (resp. (accr*)) if it satisfies (accr) (resp. (accr*)) as a module over itself.

Clearly, a module satisfies (accr*) if it satisfies (accr). We shall see that these two properties are actually equivalent.

LEMMA 2. Let $B=\left(b_{1}, b_{2}, \ldots, b_{t}\right)$ be a finitely generated ideal of a ring $R$. If $k$ and $n$ are any two positive integers such that $n>k t$, then $B^{n}=\left(b_{1}^{k}, b_{2}^{k}, \ldots, b_{t}^{k}\right) B^{n-k}$.

Proof. Put $\left(b_{1}^{k}, b_{2}^{k}, \ldots, b_{t}^{k}\right) B^{n-k}=A$. Then $A \subseteq B^{n}$ and $B^{n}$ is generated by

$$
T=\left\{b_{1}^{\lambda_{1}} b_{2}^{\lambda_{2}} \cdots b_{t}^{\lambda_{t}} ; \lambda_{i} \geq 0, \lambda_{1}+\lambda_{2}+\cdots+\lambda_{t}=n\right\} .
$$

Since $n>k t$, for each $\alpha=b_{1}^{\lambda_{1}} b_{2}^{\lambda_{2}} \cdots b_{t}^{\lambda_{t}} \in T$, there exists at least one $i$ such that $\lambda_{t}>k$. Hence, $\alpha=b_{i}^{k}\left(b_{1}^{\lambda_{1}} b_{2}^{\lambda_{2}} \cdots b_{i}^{\lambda_{t}-k} \cdots b_{t}^{\lambda_{t}}\right) \in A$ for every $\alpha \in T$ as $\lambda_{1}+\cdots+\left(\lambda_{i}-k\right)+\cdots+\lambda_{t}=n-k$, so $B^{n}=A=$ $\left(b_{1}^{k}, b_{2}^{k}, \ldots, b_{t}^{k}\right) B^{n-k}$.

THEOREM 1. For any $R$-module $M$, two properties (accr) and (accr*) of $M$ are equivalent.

Proof. It is sufficient to show that (accr*) implies (accr). So we consider the ascending chain of submodules $\left\{N: B^{k}\right\}_{k \in Z^{+}}$for any sub- 
module $N$ of $M$ and any finitely generated ideal $B=\left(b_{1}, b_{2}, \ldots, b_{t}\right)$ of $R$. Since $M$ satisfies (accr*), for each $b_{i}$ there exists an $n_{i}$ such that $N: b_{i}^{n_{i}}=N: b_{i}^{n_{i}+\jmath}$ for every $j \geq 1$. Now, let $k=\max \left\{n_{i} ; i=1,2, \ldots, t\right\}$. Then

$$
\begin{aligned}
N:\left(b_{1}^{k}, b_{2}^{k}, \ldots, b_{t}^{k}\right) & =\bigcap_{i=1}^{t}\left(N: b_{i}^{k}\right)=\bigcap_{i=1}^{t}\left(N: b_{i}^{k+j}\right) \\
& =N:\left(b_{1}^{k+j}, b_{2}^{k+j}, \ldots, b_{t}^{k+j}\right) \text { for every } j \geq 1 .
\end{aligned}
$$

Now, let $n$ be a positive integer such that $n>k t$; then by Lemma 2 we have $B^{n}=\left(b_{1}^{k}, b_{2}^{k}, \ldots, b_{t}^{k}\right) B^{n-k}$. It follows that

$$
\begin{aligned}
N: B^{n} & =\left(N:\left(b_{1}^{k}, b_{2}^{k}, \ldots, b_{t}^{k}\right)\right): B^{n-k} \\
& =\left(N:\left(b_{1}^{k+j}, b_{2}^{k+j}, \ldots, b_{t}^{k+j}\right)\right): B^{n-k} \quad \text { for every } j \geq 1 \\
& =N:\left(b_{1}^{k+j}, b_{2}^{k+j}, \ldots, b_{t}^{k+j}\right) B^{n-k} \quad \text { for every } j \geq 1 \\
& \supseteq N: B^{n+j} \quad \text { for every } j \geq 1 \\
& \supseteq N: B^{n},
\end{aligned}
$$

because $\left(b_{1}^{k+j}, b_{2}^{k+\jmath}, \ldots, b_{t}^{k+j}\right) B^{n-k} \subseteq B^{n+j} \subseteq B^{n}$.

COROllary. For any $R$-module $M$, the following statements are equivalent:

(1) M satisfies (accr);

(2) $M$ satisfies $\left(\right.$ accr* ${ }^{*}$;

(3) For any submodule $N$ of $M$ and any element $r \in R$, there exists a positive integer $n$ such that $N=\left(N: r^{h}\right) \cap\left(N+r^{h} M\right)$ for every $h \geq n$;

(4) For any submodule $N$ of $M$ and any finitely generated ideal $B$ of $R$, there exists a positive integer $n$ such that $N=\left(N: B^{h}\right) \cap\left(N+B^{h} M\right)$ for every $h \geq n$.

Proof. (1) $\Leftrightarrow$ (2) $\Leftrightarrow$ (3) follows from Theorem 1 and Proposition 1 . $(4) \Rightarrow(3)$ is obvious and (1) $\Rightarrow(4)$ can be proved in a way similar to the proof of [4], p. 178, Lemma 1.

Clearly, every Noetherian module and every module over an Artinian ring satisfy (accr). Similarly, an $R$-module $M$ which has ACC on colon submodules $L: A$, where $L$ is any submodule of $M$ and $A$ is any subset of $R$, also satisfies (accr) [14]. In [12], a ring $R$ is called an $N$-ring if, for every ideal $A$ of $R$, there exists a Noetherian ring extension $R^{\prime}$ of $R$ such that $A$ is contracted from $R^{\prime}$, i.e., $A R^{\prime} \cap R=A$. Every $N$-ring is known to be strongly Laskerian. It is also known that every finitely generated 
module over an $N$-ring has ACC on colon submodules ([14], p. 120, Theorem 2.3 and its Corollary 2.5). So every $N$-ring and every finitely generated module over an $N$-ring satisfy (accr). More generally, we have

Proposition 3. Every Laskerian module satisfies (accr).

This is a direct result of Proposition 2.

Definition 2 ([7]). A ring $R$ is called a perfect ring if every flat $R$-module is projective.

Perfect rings can be characterized in various ways. For example, a ring $R$ is perfect iff $R$ satisfies DCC on principal ideals ([7], p. 466, Theorem 3.2) iff every $R$-module $M$ satisfies ACC on submodules generated by $n$ elements for each $n \geq 1$ ([18], p. 269, Proposition 1.2). Therefore, every quasi-local ring with nilpotent maximal ideal is perfect. From these remarks we have

Proposition 4. Every module over a perfect ring satisfies (accr). In particular, every quasi-local ring with nilpotent maximal ideal satisfies (accr).

In [12], p. 129, Corollary 2.11, it was proved that a quasi-local ring $R$ whose maximal ideal has the order of nilpotency $n=2$ is an $N$-ring. This result is followed by an example of a quasi-local ring with $n=3$, which is not an $N$-ring. Therefore, in view of Proposition 4, the class of $N$-ring is indeed a proper subset of the class of rings which satisfy (accr).

2. Properties of modules satisfying (accr). In this section we consider various properties of modules satisfying (accr), in particular, those properties which are in common with Noetherian modules.

Proposition 5. Every irreducible submodule $N$ of an $R$-module $M$ satisfying (accr) is primary.

Proof. Let $r e \in N$, where $r \in R$ and $e \in M-N$. Then $N \varsubsetneqq N: r$ so that $N \varsubsetneqq N: r^{k}$ for every $k \geq 1$. By Proposition 1 , we have $N \stackrel{\ddagger}{=}\left(N: r^{h}\right)$ $\cap\left(N+r^{h} M\right)$ for a sufficiently large integer $h$. Since $N$ is irreducible, $N=N+r^{h} M$ whence $r^{h} M \subseteq N$. Hence $N$ is primary.

In [12], p. 130, Example 2.13, it was shown that a ring $R$ is not necessarily an $N$-ring even though $R / A$ is an $N$-ring for every nonzero ideal $A$ of $R$. In contrast with this, here we have

Proposition 6. An R-module $M$ satisfies (accr) if, and only if, so does a factor module $M / K$ for every non-zero submodule $K$ of $M$. 
Proof. Let $\eta$ be the natural homomorphism of $M$ onto $M / K$. If $N$ is a submodule of $M$ and $B$ is a finitely generated ideal of $R$, then $\eta(N):_{M / K} B=\left((N+K):_{M} B\right) / K$. Hence, $M / K$ satisfies (accr) if $M$ does. Conversely, we assume that there exists an ascending chain of submodules $\left\{N: B^{k}\right\}_{k \in Z^{+}}$which does not terminate, although $M / K$ satisfies (accr) for every non-zero submodule $K$. Then there exists a positive integer $k^{\prime}$ such that $N \subsetneq N: B^{k^{\prime}}$. If we let $L=N: B^{k^{\prime}}$, then $L \neq(0)$ and $\left\{L: B^{k}\right\}_{k \in Z^{+}}$does not terminate either, because it consists of all but the first $k^{\prime}$ terms of the original chain $\left\{N: B^{k}\right\}_{k \in Z^{+}}$. Therefore, without loss of generality, we may assume that $N \neq(0)$. By hypothesis, $M / N$ satisfies (accr) so that $\left\{\left(N: B^{k}\right) / N\right\}_{k \in Z^{+}}$terminates. Consequently, $\left\{N: B^{k}\right\}_{k \in Z^{+}}$terminates, which is a contradiction. Hence, $M$ satisfies (accr) if so does $M / K$ for every non-zero submodule $K$.

Proposition 7. If an $R$-module $M$ satisfies (accr), then so does the $R_{m}$-module $M_{m}$ for every maximal ideal (or multiplicatively closed set) $m$. The converse is true if $R$ is a quasi-semilocal ring.

Proof. Let $\bar{N}$ be a submodule of $M_{m}$ and $\bar{B}$ an ideal of $R_{m}$ finitely generated by $\left\{b_{1} / s_{1}, b_{2} / s_{2}, \ldots, b_{n} / s_{n}\right\}$. Put $N=\bar{N}^{c}$ and $B=$ $\left(b_{1}, b_{2}, \ldots, b_{n}\right) R$. Then $\bar{N}=N R_{m}=N_{m}$ and $\bar{B}=B R_{m}=B_{m}$. Since $B$ is finitely generated, $\bar{N}:_{M_{m}} \bar{B}^{k}=N_{m}:_{M_{m}} B_{m}^{k}=\left(N:_{M} B^{k}\right)_{m}$ for every $k \in Z^{+}$. Hence, if $M$ satisfies (accr), then $M_{m}$ also satisfies (accr) as an $R_{m}$-module. To prove the converse, let $m_{1}, m_{2}, \ldots, m_{n}$ be the maximal ideals of a quasi-semilocal ring $R$ and let $\left\{N: B^{k}\right\}_{k \in Z^{+}}$be an ascending chain for a submodule $N$ of $M$ and a finitely generated ideal $B$ of $R$. Then, for each $i=1,2, \ldots, n$, there exists a $k_{i} \in Z^{+}$such that $\left(N: B^{k_{t}}\right)_{m_{t}}=\left(N: B^{k_{i}+j}\right)_{m_{t}}$ for every $j \geq 0$. If $h=\max \left\{k_{i} ; i=1,2, \ldots, n\right\}$, then $N: B^{h}=N: B^{h+j}$ for every $j \geq 0$ due to [17], p. 164, Corollary to Proposition 18 .

Definition 3. Let $N$ be a submodule of an $R$-module $M$ and $B$ an ideal of $R$. We say that the Artin-Rees property holds for $N$ and $B$ if there exists a positive integer $n$ such that $N \cap B^{h} M \subseteq B N$ for every $h \geq n$. $M$ is called an Artin-Rees module if the Artin-Rees property holds for every submodule of $M$ and every ideal of $R$.

Recall that every finitely generated module $M$ over a Noetherian ring is an Artin-Rees module. This is due to a weak version of the Artin-Rees Lemma which is proved usually by applying primary decompositions of submodules of $M$. In the following Theorem 2, we generalize the ArtinRees Lemma (weak version) to modules which satisfy (accr); we prove it in the absence of the primary decomposition property of submodules. 
THEOREM 2. If an R-module $M$ satisfies (accr), then the Artin-Rees property holds for every submodule $N$ of $M$ and every finitely generated ideal $B$ of $R$.

Proof. Put $L=B N$. Then, there exists a positive integer $n$ such that $L=\left(L: B^{h}\right) \cap\left(L+B^{h} M\right)$ for every $h \geq n$ by the Corollary to Theorem 1. Equivalently, $\left(L: B^{h}\right) \cap B^{h} M \subseteq L$ for every $h \geq n$ by the Dedekind's modular law. Clearly, $N \subseteq L: B^{h}$. So $N \cap B^{h} M \subseteq\left(L: B^{h}\right) \cap B^{h} M \subseteq L$ $=B N$ for every $h \geq n$.

For any ideal $B$ of a ring $R$ and an $R$-module $M$, we always have (0) $\subseteq 0^{S} \subseteq B \bigcap_{n=1}^{\infty} B^{n} M \subseteq \bigcap_{n=1}^{\infty} B^{n} M$, where $S=\{1-b ; b \in B\}$ and $0^{S}=\{x \in M ; s x=0$ for some $s \in S\}$. The Krull Intersection Theorem states that if $M$ is a Noetherian $R$-module and $B$ is an ideal of $R$, then $B \bigcap_{n=1}^{\infty} B^{n} M=\bigcap_{n=1}^{\infty} B^{n} M$. Since $\bigcap_{n=1}^{\infty} B^{n} M$ is finitely generated, $\bigcap_{n=1}^{\infty} B^{n} M=0^{S}$. We shall see that the same result holds for $R$-modules $M$ satisfying (accr) and finitely generated ideals $B$ of $R$, although $\cap_{n=1}^{\infty} B^{n} M$ may not be finitely generated.

LEMMA 3. Let $B$ be an ideal of $R$ such that the Artin-Rees property holds for $B$ and any cyclic submodule of an $R$-module $M$. Then $0^{S}=$ $B \bigcap_{n=1}^{\infty} B^{n} M=\bigcap_{n=1}^{\infty} B^{n} M$.

Proof. Let $x \in \bigcap_{n=1}^{\infty} B^{n} M$. Then the cyclic submodule $R x$ is contained in $B^{n} M$, so that $R x=R x \cap B^{n} M$ for every $n \in z^{+}$. By hypothesis, $R x \cap B^{h} M \subseteq B x$ for all sufficiently large positive integers $h$. Consequently, $R x=B x$ whence there exists an element $b$ of $B$ such that $(1-b) x=0$, i.e., $x \in 0^{S}$. Hence, $0^{S}=B \cap_{n=1}^{\infty} B^{n} M=\bigcap_{n=1}^{\infty} B^{n} M$.

Combining Theorem 2 and Lemma 3, we have

THEOREM 3. Let $M$ be an $R$-module which satisfies (accr). Then $0^{S}=B \cap_{n=1}^{\infty} B^{n} M=\cap_{n=1}^{\infty} B^{n} M$ for every finitely generated ideal $B$ of $R$. Consequently, if either $B$ is a finitely generated ideal contained in the Jacobson radical of $R$ or $R$ is an integral domain over which $M$ is torsion free, then $\cap_{n=1}^{\infty} B^{n} M=(0)$.

The following Corollary to Theorem 3 is a slight improvement of [13], p. 109, Corollary 3.2, which follows from Proposition 3 and Theorem 3.

COROLlARY. If $M$ is a Laskerian $R$-module and $B$ is a finitely generated ideal contained in the Jacobson radical of $R$, then $\bigcap_{n=1}^{\infty} B^{n} M=(0)$. 
In Proposition 3 we have seen that every Laskerian module satisfies (accr). It is natural to ask if there exists a module which satisfies (accr), but is not a Laskerian module. The answer to this question is affirmative as we shall see in the following example.

EXAMPLE 1. Let $M=\sum \oplus_{p \in \Lambda} Z /(p)$ be a module over the ring $Z$ of integers, where $\Lambda$ is the set of all prime integers of $Z$. For each $\bar{e} \in M$, we write $\bar{e}=\sum_{p \in \Lambda} \bar{e}_{p}$, where $\bar{e}_{p}=e_{p}+(p) \in Z /(p)$ and $e_{p} \in Z$. Since the set of zero divisors of $M$ is identical with $\bigcup_{p \in \Lambda}(p), M$ is not a $Z D$-module. Consequently, $M$ is not Laskerian (cf. [13], p. 102). Next, let $N$ be a submodule of $M$. Then it has the form $N=\sum \oplus_{p \in S} Z /(p)$ for some $S \subseteq \Lambda$ (cf. [10], p. 367, Example 2). Let $r \in Z$ and $k \in Z^{+}$. Then $\bar{e}=\sum_{p \in \Lambda} \bar{e}_{p} \in N: r^{k}$ iff $r^{k} \bar{e}=\sum_{p \in \Lambda} r^{k} \bar{e}_{p} \in N$ iff $r^{k} \bar{e}_{p}=0$ of $Z /(p)$ for every $p \notin S$ iff $p \mid\left(r^{k} e_{p}\right)$ for every $p \notin S$ iff $p \mid r$ or $p \mid e_{p}$ for every $p \notin S$ iff $p \mid r e_{p}$ for every $p \notin S$ iff $r \bar{e}_{p}=0$ of $Z /(p)$ for every $p \notin S$ iff $\bar{e}=\sum_{p \in \Lambda} \bar{e}_{p} \in N: r$. Thus $N: r=N: r^{k}$ for every $k \in Z^{+}$and so $M$ satisfies (accr). According to Theorem 2, $M$ is an Artin-Rees module. We note that every submodule $N$ of $M$ is actually a pure submodule, i.e., $N \cap a M=a N$ for every $a \in Z$.

Every module over an Artinian ring satisfies (accr). In the following Example 2, we show that not every module over a Noetherian ring satisfies (accr).

EXAMPLE 2. Let $V$ be a countably infinite dimensional vector space over a field $F$ with a basis $D=\left\{v_{1}, v_{2}, \ldots, v_{i}, \ldots\right\}$. For each positive integer $i$, let $V_{i}$ be the subspace of $V$ generated by $\left\{v_{1}, v_{2}, \ldots, v_{i}\right\}$. Define the linear transformation $T$ on $V$ by $T\left(v_{1}\right)=0$ and $T\left(v_{i}\right)=v_{l-1}$ for every $i \geq 2$. Let $F[T]$ be the polynomial ring generated by $T$ over $F$. Then $F[T]$ is a Noetherian ring with identity. Consider $V$ as an $F[T]$-module. Now, applying the facts that $T^{k}\left(v_{j}\right)=v_{J-k}$ if $j>k$ and $T^{k}\left(v_{J}\right)=0$ otherwise, and that $D$ is linearly independent over $F$, we can prove that (0) $:{ }_{V} T^{k}=V_{k}$ for every positive integer $k$. Therefore, the sequence $(0):(T) \subseteq(0):\left(T^{2}\right) \subseteq(0):\left(T^{3}\right) \subseteq \cdots$ does not terminate. Hence, the $F[T]$-module $V$ does not satisfy (accr) and so it is not a Laskerian module by Proposition 3. More precisely, (0) has no primary decomposition due to Proposition 2.

The modules used in the above Example 1 and Example 2 appeared in [10] and [9], respectively, for other contexts.

3. Some special rings and modules satisfying (accr). In [1], Theorem 4.1 is concerned with quasi-local rings $R$ with finitely generated maximal ideals. The theorem lists five conditions, each of which is 
equivalent to that $R$ is Noetherian. As remarked in p. 21 of [1], this result can be generalized to quasi-semilocal rings in which all maximal ideals are finitely generated. In the next theorem, we improve this theorem further by adding few more equivalent conditions and give a simple proof using results of previous sections. The added conditions $((3),(4),(6))$ are either weaker or simpler than those stated in Theorem 4.1 of [1], if $R$ is an ordinary ring.

THEOREM 4. Let $R$ be a quasi-semilocal ring whose Jacobson radical $J$ is finitely generated. Then the following statements are equivalent:

(1) $R$ is a Noetherian ring;

(2) Every finitely generated ideal of $R$ has a primary decomposition;

(3) The ascending chain of ideals $\left\{A: r^{k}\right\}_{k \in Z^{+}}$terminates for every $r \in R$ and every finitely generated ideal $A$ of $R$;

(4) For any finitely generated ideal $A$ and $r \in R$, there exists a positive integer $n$ such that $A=\left(A: r^{h}\right) \cap\left(A+r^{h} R\right)$ for every $h \geq n$;

(5) For any two finitely generated ideals $A$ and $B$ of $R$, there exists $a$ positive integer $n$ such that $A=\left(A: B^{h}\right) \cap\left(A+B^{h}\right)$ for every $h \geq n$;

(6) For any two finitely generated ideals $A$ and $B$ of $R$, there exists $a$ positive integer $n$ such that $A \cap B^{h} \subseteq A B$ for every $h \geq n$, i.e., the ArtinRees property holds for $A$ and $B$;

(7) $\cap_{n=1}^{\infty} J^{n} M=(0)$ for every finitely generated $R$-module $M$;

(8) $\cap_{n=1}^{\infty}\left(A+J^{n}\right)=A$ for every finitely generated ideal $A$;

(9) For any ideal $B$ and any finitely generated ideal $A$ of $R, B=A+J B$ implies $A=B$.

Proof. (1) $\Rightarrow(2)$ is trivially true and $(1) \Rightarrow(7) \Rightarrow(8)$ is well-known. (2) $\Rightarrow(3) \Leftrightarrow$ (4) follows from Proposition 2 and Proposition 1, respectively. (4) $\Leftrightarrow(5) \Rightarrow(6)$ can be proved by applying Corollary to Theorem 1 and Theorem 2, respectively, after making suitable modification on finiteness of generating sets of submodules in the theorems. To show $(6) \Rightarrow(8)$, let $A$ be a finitely generated ideal of $R$ and consider $\bar{R}=R / A$ as an $R$-module. By (6), we can see easily that the Artin-Rees property also holds for every finitely generated submodule of $\bar{R}$ and every finitely generated ideal of $R$. Now, applying Lemma 2 and the fact that $J$ is finitely generated, we have that the $R$-module $\bar{R}$ is a Hausdorff space for the $J$-adic topology. Therefore, $\bigcap_{n=1}^{\infty}\left(A+J^{n}\right)=A$. As for $(8) \Rightarrow(9)$, we can see that $B=A+J B$ implies $B=A+J^{n} B$ for every $n>0$, hence $B=\bigcap_{n=1}^{\infty}\left(A+J^{n} B\right) \subseteq \bigcap_{n=1}^{\infty}\left(A+J^{n}\right)=A \subseteq B$. Since $(9) \Rightarrow$ (1) was already proved in [1], the proof of the theorem is completed. 
We remark that among the statements in Theorem 6, we always have the implications $(1) \Rightarrow(2) \Rightarrow(3) \Leftrightarrow(4) \Leftrightarrow(5) \Rightarrow(6)$ for any ring $R$ which may not be a quasi-semilocal ring.

COROLlARY. Let $R$ be a ring which satisfies (accr). If every maximal ideal of $R$ is finitely generated, then $R$ is locally Noetherian, that is, $R_{m}$ is Noetherian for every maximal ideal $m$ of $R$; consequently, $R$ satisfies $A C C$ for prime ideals.

A module $M$ over an associative ring $S$ (with or without identity) is said to be finite dimensional over $S$ if there does not exist an infinite chain of submodules of $M$ of the form $M_{1} \subset M_{1} \oplus M_{2} \subset M_{1} \oplus M_{2} \oplus M_{3}$ $\subset \cdots$, where each $M_{i}$ is a nonzero submodule of $M$ [10]. It is easy to see that Noetherian modules and Artinian modules are finite dimensional. However, a finite dimensional module is not necessarily Noetherian or Artinian.

Next, we consider any associative (commutative or non-commutative) ring $R$ with identity which satisfies the ascending chain condition for ideals. If $R$ is commutative, then clearly it is Laskerian. However, it is known that in the non-commutative case $R$ may satisfy the ACC for ideals without being a Laskerian ring. In view of this, P. J. McCarthy [16] proved that the following conditions are equivalent for any ring $R$ with identity, which satisfies the ACC for ideals: (i) $R$ is a Laskerian ring; (ii) $R$ is an Artin-Rees ring; (iii) If $A$ and $B$ are ideals of $R$, then $A=$ $\left(A: B^{n}\right) \cap\left(A+B^{n}\right)$ for all large integers $n$, i.e., $R$ satisfies (accr).

Now, we consider a theorem, which is similar to the above mentioned theorem of McCarthy, for finite dimensional modules.

In the rest of this paper, as we assumed at the beginning, every ring is a commutative ring with identity.

Let $M$ be an $R$-module such that each factor module of $M$ is finite dimensional. According to Theorem 2.6 of [10], p. 364, a sufficient condition for this module $M$ to be Laskerian is that $M$ is an Artin-Rees module. From the proofs of [10], Theorem 2.6 and its Lemmas 2.1 and 2.3, we can see that this condition can be replaced with a weaker condition $(\alpha)$ : the Artin-Rees property holds for every submodule of $M$ and every principal ideal of $R$. In the next theorem, we see that $(\alpha)$ is also a necessary condition for $M$ to be Laskerian.

THEOREM 5. Let $M$ be an $R$-module such that each factor module of $M$ is finite dimensional. Then the following statements are equivalent:

(1) $M$ is Laskerian; 
(2) M satisfies (accr);

(3) The Artin-Rees property holds for every submodule of $M$ and every principal (or finitely generated) ideal of $R$.

Proof. Clearly we have that $(1) \Rightarrow(2) \Rightarrow(3)$ by Proposition 3 and Theorem 2 , and (3) $\Rightarrow(1)$ follows from the above remark.

4. Topological rings satisfying (accr). Let $I=\left(a_{1}, a_{2}, \ldots, a_{n}\right)$ be an ideal of a ring $R$ such that $\bigcap_{k=1}^{\infty} I^{k}=(0)$ and $R^{*}$ the completion of $R$ for the $I$-adic topology. It is well-known that

$$
R^{*} \cong R\left[\left[x_{1}, \ldots, x_{n}\right]\right] /\left(x_{1}-a_{1}, \ldots, x_{n}-a_{n}\right)^{*},
$$

where $x_{1}, \ldots, x_{n}$ are indeterminates and $\left(x_{1}-a_{1}, \ldots, x_{n}-a_{n}\right) *$ is the closure of the ideal $H=\left(x_{1}-a_{1}, \ldots, x_{n}-a_{n}\right)$ of $R\left[\left[x_{1}, \ldots, x_{n}\right]\right]$ for the $\left(a_{1}, \ldots, a_{n}, x_{1}, \ldots, x_{n}\right)$-adic topology. In particular, if $R$ is Noetherian, then $R^{*} \cong R\left[\left[x_{1}, \ldots, x_{n}\right]\right] /\left(x_{1}-a_{1}, \ldots, x_{n}-a_{n}\right)$. In [2], p. 135, Corollary 1.3, it was pointed out that $H^{*}$ is identical with the closure of $H$ for the $\left(x_{1}, \ldots, x_{n}\right)$-adic topology of $R\left[\left[x_{1}, \ldots, x_{n}\right]\right]$ for any ring $R$.

In this section, we study those rings $R$ with (accr) which have the isomorphism $R^{*} \cong R\left[\left[x_{1}, \ldots, x_{n}\right]\right] /\left(x_{1}-a_{1}, \ldots, x_{n}-a_{n}\right)$. We focus our attention on rings of (Krull) dimension $\leq 1$. This study will be continued to the next section for coherent rings.

The following Lemma 4 for Theorem 6 is a combined form of Lemma 3.1 and Theorem 3.3, both of [2].

LEMMA 4. Let a be an element of $R$ such that $x-a$ is regular in $R[[x]]$. Then the following two statements are equivalent:

(1) The ascending chain of ideals $\left\{\text { Ann } a^{k}\right\}_{k \in Z^{+}}$terminates;

(2) In $R[[x]]$, the ideal $(x-a)$ is a subspace for the $(x)$-adic topology.

COROLlaRY. Let $R$ be a ring satisfying (accr), a an element of $R$ such that $\cap_{k=1}^{\infty}\left(a^{k}\right)=(0)$, and $R^{*}$ the completion of $R$ for the (a)-adic topology. Then the ideal $(x-a)$ of $R[[x]]$ is closed for the $(x)$-adic topology. Hence, $R^{*} \cong R[[x]] /(x-a)(c f .[2], p .143$, Theorem 3.5).

THEOREM 6. If $R$ is either a zero-dimensional ring or a one-dimensional domain, then $R$ satisfies (accr).

Proof. Assume that $\operatorname{dim} R=0$. Then, for every $a \in R, x-a$ is regular in $R[[x]]$ and the ideal $(x-a)$ contains a power of $x$ due to [11], Proposition 2.1 and [11], Proposition 2.4, respectively. Accordingly, 
$(x-a)$ is a subspace of $R[[x]]$ for the $(x)$-adic topology, whence the ascending chain of ideals $\left\{\text { Ann } a^{k}\right\}_{k \in Z^{+}}$terminates by Lemma 4. Next, let $I$ be any proper ideal of $R$. Then $R / I$ is also a zero-dimensional ring. Applying the first part of this proof, we can see that the ascending chain of ideals $\left\{\text { Ann } \bar{a}^{k}\right\}_{k \in Z^{+}}$of $R / I$ terminates for every $\bar{a}=a+I \in R / I$. This means that the ascending chain of ideals $\left\{I: a^{k}\right\}_{k \in Z^{+}}$of $R$ terminates, so $R$ satisfies (accr). Finally, we assume that $R$ is a one-dimensional domain. Then $R / I$ is a zero-dimensional ring for every non-zero ideal $I$, so that $R$ satisfies (accr) as we have seen above.

An integral domain $R$ is called a pseudo-valuation domain (PVD) if $R$ has a valuation overring $V$ such that $\operatorname{Spec}(R)=\operatorname{Spec}(V)$ as sets [8]. It is known that every PVD is a quasi-local domain and that if a PVD $R$ is not a valuation domain, then $R$ is coherent iff its unique maximal ideal is finitely generated.

Corollary. Let $R$ be a coherent PVD which is not a valuation domain. Then $R$ is Noetherian if, and only if, it satisfies (accr). Consequently, every one-dimensional coherent PVD is either Noetherian or a valuation domain ([8], p. 563, Remark 4.6).

Proof. The corollary follows from Theorem 4 and Theorem 6.

THEOREM 7. If $R$ is a zero-dimensional ring and $I=\left(a_{1}, a_{2}, \ldots, a_{n}\right)$ is an ideal of $R$ such that $\bigcap_{k=1}^{\infty} I^{k}=(0)$, then the completion $R^{*}$ of $R$ for the I-adic topology is isomorphic to $R\left[\left[x_{1}, \ldots, x_{n}\right]\right] /\left(x_{1}-a_{1}, \ldots, x_{n}-a_{n}\right)$.

Proof. By [11], Proposition 2.4, the ideal $\left(x_{i}-a_{i}\right)$ of $R\left[\left[x_{i}\right]\right]$ contains a power of $x_{l}$ for every $i=1,2, \ldots, n$. Hence the ideal $H=$ $\left(x_{1}-a_{1}, \ldots, x_{n}-a_{n}\right)$ of $R\left[\left[x_{1}, \ldots, x_{n}\right]\right]$ contains a power of $\left(x_{1}, \ldots, x_{n}\right)$, so that $H$ is an open and closed ideal for the $\left(x_{1}, \ldots, x_{n}\right)$-adic topology. Therefore, $R^{*} \cong R\left[\left[x_{1}, \ldots, x_{n}\right]\right] /\left(x_{1}-a_{1}, \ldots, x_{n}-a_{n}\right)$.

5. Topological coherent rings satisfying (accr). We have seen generalizations of both a weak version of the Artin-Rees Lemma and the Krull Intersection Theorem to modules satisfying (accr) in Theorem 2 and Theorem 3, respectively. These results imply other Noetherian properties to this class of modules when they are considered as topological modules.

Proposition 8. If $M$ is an R-module satisfying (accr) and $B$ is a finitely generated ideal contained in $J(R)$, then every submodule $N$ of $M$ is closed for the B-adic topology. 
Proof. Put $\bar{M}=M / N, \operatorname{Ann}(M / N)=I$, and $\bar{R}=R / I$. Then the $\bar{R}$-module $\bar{M}$ satisfies (accr) by Proposition 6. Since $\bar{B}=(B+I) / I$ is a finitely generated ideal contained in $J(\bar{R}), \bigcap_{n=1}^{\infty} \bar{B}^{n} \bar{M}=(\overline{0})$ by Theorem 3. Thus we have $\bigcap_{n=1}^{\infty}\left(N+B^{n} M\right)=N$.

Proposition 9. Let $R$ be a ring satisfying (accr) and $B$ a finitely generated ideal of $R$ contained in $J(R)$. Then each ideal of $R$ is the contraction of an ideal of the completion $R^{*}$ of $R$ for the B-adic topology. Consequently, if $R^{*}$ is Noetherian, then so is $R$.

Proof. Apply Proposition 8 and [15], p. 292, Theorem 1.

LEMMA 5. Let $M$ be an $R$-module satisfying (accr), $E$ an $R$-module, and $B$ a finitely generated ideal of $R$. Then each homomorphism $f: E \rightarrow M$ is a strict morphism for the B-adic topologies.

Proof. Refer to the proof of [6], p. 64, Corollary to Theorem 2 and apply Theorem 2 .

Proposition 10. Let $L \stackrel{f}{\rightarrow} M \stackrel{g}{\rightarrow} N$ be an exact sequence of $R$-modules, where $M$ and $N$ satisfy (accr), and let $B$ be a finitely generated ideal of $R$. If now the Hausdorff completions are taken with respect to the B-adic topologies, then $L^{*} \stackrel{f^{*}}{\rightarrow} M^{*} \stackrel{g^{*}}{\rightarrow} N^{*}$ is an exact sequence of $R^{*}$-modules.

Proof. Both $f$ and $g$ are strict morphisms by Lemma 5. Hence, the proposition follows from [6], p. 50, Lemma 2.

COROllaRY. Let $M$ be an $R$-module satisfying (accr) and $B$ a finitely generated ideal of $R$. Let $0 \rightarrow L \stackrel{f}{\rightarrow} M \stackrel{g}{\rightarrow} N \rightarrow 0$ be an exact sequence of $R$-modules. If the Hausdorff completions are taken with respect to the $B$-adic topologies, then $0 \rightarrow L^{*} \stackrel{f^{*}}{\rightarrow} M^{*} \stackrel{g^{*}}{\rightarrow} N^{*} \rightarrow 0$ is an exact sequence of $R^{*}$ modules.

Let $R$ be a topological ring with a basis $Q$ of neighborhoods of 0 consisting of ideals and let $M$ be an $R$-module equipped with the $Q$-topology. We consider the following condition (P): For every finitely generated submodule $N$ of $M$, the $Q$-topology of $N$ is equivalent to the topology induced on $N$ by the $Q$-topology of $M$. 
Definition 4. A linear topology of a ring $R$ is called an Artin-Rees topology if every finitely presented $R$-module satisfies condition (P) ([3]).

If $R$ is a ring satisfying (accr) and $B$ is a finitely generated ideal of $R$, then clearly the $B$-adic topology of the $R$-module $R$ fulfills condition (P) by Theorem 2 .

We remark that if $R$ is a coherent ring equipped with a linear topology $T$, then $T$ is an Artin-Rees topology if, and only if, the $R$-module $R$ satisfies condition (P) ([3], p. 1210, Proposition 5). Therefore, the $B$-adic topology of a coherent ring $R$ which satisfies (accr) is an Artin-Rees topology for every finitely generated ideal $B$ of $R$.

N. Radu proved ([19], Propositoin 3) that the completion of a coherent Laskerian ring $R$ for the $m$-adic topology, where $m$ is a finitely generated ideal of $R$, is a flat $R$-module. In the next theorem, we generalize this result by Radu to coherent rings satisfying (accr).

THEOREM 8. Let $R$ be a coherent ring which satisfies (accr), $B$ a finitely generated ideal of $R$, and $M$ an $R$-module. Let $R^{*}$ and $M^{*}$ be the Hausdorff completions of $R$ and $M$ for the $B$-adic topologies, respectively.

(1) If $M$ admits a finite presentation, then $R^{*} \otimes M \cong M^{*}$,

(2) $R^{*}$ is a flat $R$-module, and

(3) $R^{*}$ is a faithfully flat $R$-module if, and only if, $B$ is contained in $J(R)$.

Proof. (1): By hypothesis, $M$ admits a finite presentation $E \stackrel{f}{\rightarrow} L \stackrel{g}{\rightarrow} M \rightarrow 0$, where $E$ and $L$ are finitely generated free $R$-modules. Since the $B$-adic topology of $R$ is an Artin-Rees topology as we remarked previously, $f$ and $g$ are strict morphisms. Hence, this exact sequence of $R$-modules can be transformed to the exact sequence $E^{*} \stackrel{f^{*}}{\rightarrow} L^{*} \stackrel{g^{*}}{\rightarrow} M^{*} \rightarrow 0$ by [6], p. 50, Lemma 2. Now, we can show that $R^{*} \otimes M \cong M^{*}$ by applying the same arguments used in the proof of Theorem 3, (ii) in [6], p. 69. Clearly, (2) follows from (1) and (3) follows from (2) and Proposition 9.

By virtue of Theorem 8, now we have the following generalization of Corollary to Theorem 3 in [6], p. 69.

Corollary 1. Let $R$ and $B$ be as in Theorem 8 and $M$ an $R$-module with finite presentation. Let $E$ and $F$ be finitely generated submodules of $M$. If $R, M, E$, and $F$ are equipped with the B-adic topologies, respectively, then

(1) $E^{*}=R^{*} i(E)$, where $i$ is the canonical map of $M$ to $M^{*}$, 
(2) $(E+F)^{*}=E^{*}+F^{*}$,

(3) $(E \cap F)^{*}=E^{*} \cap F^{*}$,

(4) $(E: F)^{*}=E^{*}: F^{*}$,

(5) If $I$ and $J$ are finitely generated ideals of $R$, then $(I J)^{*}=I^{*} J^{*}$,

(6) If a is a regular element of $R$, then it is also regular in $R^{*}$.

COROllary 2. Let $R$ and $B$ be as in Theorem 8. If $\cap_{n=1}^{\infty} B^{n}=(0)$, then

(1) the ascending chain of ideals $\left\{I R^{*}: r^{k}\right\}_{k \in Z^{+}}$of $R^{*}$ terminates for every ideal $I$ of $R$ and every element $r \in R$,

(2) the Artin-Rees property holds for finitely generated extended ideals in $R^{*}$, that is, for every pair of finitely generated ideals $I$ and $J$ of $R$, there exists an $n \in Z^{+}$such that $I R^{*} \cap J^{h} R^{*} \subseteq(I J) R^{*}$ for every $h \geq n$,

(3) $I R^{*}$ is a closed ideal of $R^{*}$ for every finitely generated ideal I of $R$.

Proof. (1) is true due to the fact that $R^{*}$ is a flat $R$-module (cf. [5], p. 65, Ex. 22) and (2) is a combined result of Theorem 2 and (3) of Corollary 1 to Theorem 8. Finally, (3) follows from that $I R^{*}=I^{*}$ and that $I R^{*}$ is a subspace of $R^{*}$ for the $B$-adic topology by (2).

THEOREM 9. Let $R$ be a coherent ring satisfying (accr), $B=$ $\left(b_{1}, b_{2}, \ldots, b_{n}\right)$ an ideal of $R$ such that $\bigcap_{k=1}^{\infty} B^{k}=(0)$, and $B_{i}=B-\left\{b_{i}\right\}$ for every $i=1,2, \ldots, n$. Let $R^{*}$ and $R_{i}^{*}$ be, respectively, the completions of $R$ for the $B$-adic topology and the $B_{i}$-adic topology. Then $R^{*} \cong$ $R\left[\left[x_{1}, \ldots, x_{n}\right]\right] /\left(x_{1}-b_{1}, \ldots, x_{n}-b_{n}\right)$ if any of the following two conditions is satisfied:

(i) $\cap_{k=1}^{\infty} b_{i}^{k} R_{i}^{*}=(0)$ for every $i=1,2, \ldots, n$,

(ii) $B_{i}^{k}$ is closed for the $\left(b_{i}\right)$-adic topology of $R$ for every $k \in Z^{+}$and every $i=1,2, \ldots, n$.

Proof. According to Corollary 2 to Theorem 8, the ascending chain of ideals $\left\{\mathrm{Ann}_{R_{i}^{*}} b_{i}^{k}\right\}_{k \in Z^{+}}$in $R_{i}^{*}$ terminates for every $i$; furthermore, the ideal $b_{l}^{k} R_{i}^{*}$ is closed for the $B_{i}$-adic topology of $R_{i}^{*}$ for every $k$ and $i$. Now applying Theorem 5.1 of [2], p. 150, we can conclude that $R^{*} \cong$ $R\left[\left[x_{1}, \ldots, x_{n}\right]\right] /\left(x_{1}-b_{1}, \ldots, x_{n}-b_{n}\right)$ if condition (i) is satisfied. Similarly, the isomorphism exists if condition (ii) is satisfied because condition (ii) implies condition (i) by [2], p. 152, Lemma 5.3.

We remark that Theorem 9 is trivially true for $n=1$ in view of Corollary to Lemma 4. 
COROLlaRY. Let $R$ be a coherent ring satisfying (accr) and let $B=$ $\left\{b_{1}, b_{2}, \ldots, b_{n}\right\}$ be an ideal contained in $J(R)$. Then $R^{*} \cong$ $R\left[\left[x_{1}, \ldots, x_{n}\right]\right] /\left(x_{1}-b_{1}, \ldots, x_{n}-b_{n}\right)$.

Proof. Since $B \subseteq J(R), \bigcap_{k=1}^{\infty} B^{k}=(0)$ by Theorem 3. Moreover, condition (ii) of Theorem 9 is fulfilled due to Proposition 8.

\section{REFERENCES}

[1] D. D. Anderson, J. Matijevic and W. Nichols, The Krull intersection Theorem II, Pacific J. Math., 66, No. 1 (1976), 15-22.

[2] D. Arezzo and L. Robbiano, Sul completato di un anello rispetto ad un ideale di tipo finito, Rend. Sem. Mat. Univ. Padova, 44 (1970), 133-154.

[3] B. Ballet, Topologies linéaires sur un anneau cohérent, C. R. Acad. Sci. Paris Sér. A-B, 270 (1970), A1209-1211.

[4] W. E. Barnes and W. M. Cunnea, Ideal decompositions in Noetherian rings, Canad. J. Math., 17 (1965), 178-184.

[5] N. Bourbaki, Algèbre Commutative, Chap. 1, 2, Hermann, Paris, 1961.

[6] _ Algèbre Commutative, Chap. 3, 4, Hermann, Paris, 1961.

[7] S. U. Chase, Direct products of modules, Trans. Amer. Math. Soc., 97 (1960), 457-473.

[8] D. E. Dobbs, Coherence, ascent of going-down, and pseudo-valuation domains, Houston J. Math., 4, No. 4 (1978), 551-567.

[9] J. W. Fisher, Decomposition theories for modules, Trans. Amer. Math. Soc., 145 (1969), 241-269.

[10] _ _ The primary decomposition theory for modules, Pacific J. Math., 35, No. 2 (1970), 359-369.

[11] R. Gilmer, A. Grams and T. Parker, Zero divisors in power series rings, J. Reine Angew. Math., 278 (1975), 145-164.

[12] R. Gilmer and W. Heinzer, Ideals contracted from a Noetherian extension ring, J. Pure Appl. Algebra, 24 (1982), 123-144.

[13] W. Heinzer and D. Lantz, The Laskerian property in commutative rings, J. Algebra, 72 (1981), 101-114.

[14] __ N-rings and ACC on colon ideals, J. Pure Appl. Algebra, 32 (1984), 115-127.

[15] C. P. Lu, Purity of linearly topological rings in overrings, J. Algebra, 59, No. 2 (1979), 290-301.

[16] P. J. McCarthy, Note on primary ideal decompositions, Canad. J. Math., 18 (1966), 950-952.

[17] D. G. Northcott, Lessons on Rings, Modules and Multiplicities, Cambridge University Press, 1968.

[18] G. Renault, Sur des conditions de chaines ascendantes dans des modules libres, J. Algebra, 47 (1977), 268-275.

[19] N. Radu, Sur les anneaux cohérents Laskeriens, Rev. Roum. Math. Pures Appl., 11 (1966), 865-867.

Received September 23, 1986. 


\section{PACIFIC JOURNAL OF MATHEMATICS EDITORS}

\author{
V. S. VARADARAJAN \\ (Managing Editor) \\ University of California \\ Los Angeles, CA 90024 \\ HERBERT ClEMENS \\ University of Utah \\ Salt Lake City, UT 84112 \\ R. FINN \\ Stanford University \\ Stanford, CA 94305
}

\author{
HERMANN FLASCHKA \\ University of Arizona \\ Tucson, AZ 85721
}

RAMESh A. GANGolli

University of Washington Seattle, WA 98195

VAUGHAN F. R. JONES

University of California

Berkeley, CA 94720

\author{
ROBION KIRBY \\ University of California \\ Berkeley, CA 94720
}

C. C. MOORE

University of California

Berkeley, CA 94720

HAROLD STARK

University of California, San Diego

La Jolla, CA 92093

\section{ASSOCIATE EDITORS}
R. AREnS
E. F. BECKENBACH
B. H. NEUMANN
F. WOLF
K. YOSHIDA

(1906-1982)

\section{SUPPORTING INSTITUTIONS}

\begin{abstract}
UNIVERSITY OF ARIZONA
UNIVERSITY OF BRITISH COLUMBIA

CALIFORNIA INSTITUTE OF TECHNOLOGY

UNIVERSITY OF CALIFORNIA

MONTANA STATE UNIVERSITY

UNIVERSITY OF NEVADA, RENO

NEW MEXICO STATE UNIVERSITY

OREGON STATE UNIVERSITY
\end{abstract}

\author{
UNIVERSITY OF OREGON \\ UNIVERSITY OF SOUTHERN CALIFORNIA \\ STANFORD UNIVERSITY \\ UNIVERSITY OF HAWAII \\ UNIVERSITY OF TOKYO \\ UNIVERSITY OF UTAH \\ WASHINGTON STATE UNIVERSITY \\ UNIVERSITY OF WASHINGTON
}

The Supporting Institutions listed above contribute to the cost of publication of this Journal, but they are not owners or publishers and have no responsibility for its content or policies.

Mathematical papers intended for publication in the Pacific Journal of Mathematics should be in typed form or offset-reproduced (not dittoed), double spaced with large margins. Please do not use built up fractions in the text of the manuscript. However, you may use them in the displayed equations. Underline Greek letters in red, German in green, and script in blue. The first paragraph must be capable of being used separately as a synopsis of the entire paper. In particular it should contain no bibliographic references. Please propose a heading for the odd numbered pages of less than 35 characters. Manuscripts, in triplicate, may be sent to any one of the editors. Please classify according to the scheme of Math. Reviews, Index to Vol. 39. Supply name and address of author to whom proofs should be sent. All other communications should be addressed to the managing editor, or Elaine Barth, University of California, Los Angeles, California 90024.

There are page-charges associated with articles appearing in the Pacific Journal of Mathematics. These charges are expected to be paid by the author's University, Government Agency or Company. If the author or authors do not have access to such Institutional support these charges are waived. Single authors will receive $\mathbf{5 0}$ free reprints; joint authors will receive a total of $\mathbf{1 0 0}$ free reprints. Additional copies may be obtained at cost in multiples of 50 .

The Pacific Journal of Mathematics is issued monthly as of January 1966. Regular subscription rate: $\$ 190.00$ a year (5 Vols., 10 issues). Special rate: $\$ 95.00$ a year to individual members of supporting institutions.

Subscriptions, orders for numbers issued in the last three calendar years, and changes of address should be sent to Pacific Journal of Mathematics, P.O. Box 969, Carmel Valley, CA 93924, U.S.A. Old back numbers obtainable from Kraus Periodicals Co., Route 100, Millwood, NY 10546.

The Pacific Journal of Mathematics at P.O. Box 969, Carmel Valley, CA 93924 (ISSN 0030-8730) publishes 5 volumes per year. Application to mail at Second-class postage rates is pending at Carmel Valley, California, and additional mailing offices. Postmaster: send address changes to Pacific Journal of Mathematics, P.O. Box 969, Carmel Valley, CA 93924.

PUBLISHED BY PACIFIC JOURNAL OF MATHEMATICS, A NON-PROFIT CORPORATION Copyright (C) 1988 by Pacific Journal of Mathematics 


\section{Pacific Journal of Mathematics}

\section{Vol. 131, No. 2 December, 1988}

Selman Akbulut and Henry Churchill King, Polynomial equations of immersed surfaces .................................... 209

Alberto Baider and Richard C. Churchill, The Campbell-Hausdorff group and a polar decomposition of graded algebra automorphisms ........2 219

Wayne C. Bell and John William Hagood, Separation properties and exact

Radon-Nikodým derivatives for bounded finitely additive measures . . . 237

Dennis J. Garity, James P. Henderson and David G. Wright, Menger

spaces and inverse limits ...............................249

B. Brent Gordon, Algebraically defined subspaces in the cohomology of a

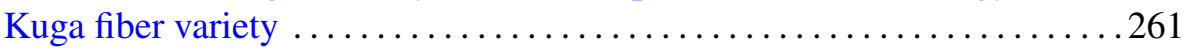

Jeffrey A. Hogan, Weighted norm inequalities for the Fourier transform on connected locally compact groups ........................... 277

Guojun Liao, A study of regularity problem of harmonic maps ..........291

Chin-pi Lu, Modules satisfying ACC on a certain type of colons ......... 303

Kunio Murasugi, Jones polynomials of periodic links

Hans Schoutens, Approximation properties for some non-Noetherian local

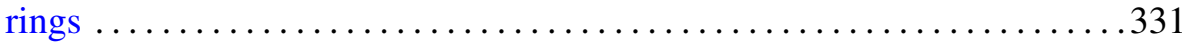

Peter Sjögren, Convergence for the square root of the Poisson kernel ...... 361 Alexandru Ion Suciu, The oriented homotopy type of spun 3-manifolds .393 